\title{
Sporadic colonic polyposis and adenocarcinoma associated with lymphoblastic and large B-cell lymphoma in a young male patient: A case report
}

\author{
SEYED MEHDI HASHEMI ${ }^{1,2}$, SEYED AMIRHOSSEIN FAZELI ${ }^{3}$, \\ FATEMEH ARABPOUR-DAHOUEI ${ }^{4}$, ALI DAVARIAN ${ }^{5,6}$ and REZA GOLABCHIFARD ${ }^{3}$ \\ ${ }^{1}$ Division of Hematology and Medical Oncology, Department of Internal Medicine, School of Medicine,
} Zahedan University of Medical Sciences; ${ }^{2}$ Division of Hematology and Medical Oncology, Department of Internal Medicine, Ali-Ebne-Abitaleb Hospital; ${ }^{3}$ Department of Internal Medicine, School of Medicine; ${ }^{4}$ Students' Scientific Research Center, Zahedan University of Medical Sciences, Zahedan 9816743111, Iran; ${ }^{5}$ Department of Biochemistry and Molecular Biophysics, School of Medicine, Washington University, St. Louis, MO 63130, USA; ${ }^{6}$ Ischemic Disorders Research Center,

Golestan University of Medical Sciences, Gorgan 4914815855, Iran

Received October 1, 2015; Accepted December 3, 2015

DOI: $10.3892 / \mathrm{mco} .2015 .718$

\begin{abstract}
We herein report a case of colonic polyposis, colorectalcarcinomaandlarge B-cell lymphomaina 22-year-old male patient with a previous history of childhood lymphoblastic lymphoma. Eight years after lymphoblastic lymphoma, which presented as mediastinal mass and superior vena cava syndrome, the patient complained of abdominal pain, lower gastrointestinal bleeding and an abdominal mass. The surgical exploration revealed numerous mucosal polyps throughout the large intestine, and multifocal masses in the ascending and transverse colon and the rectosigmoid region. A retroperitoneal mass was also found. The pathological examination revealed $>100$ tubular adenomatous polyps and a multifocal, well-differentiated adenocarcinoma, with lymph node involvement and pericolic invasion. Interestingly, the immunohistochemical studies confirmed the malignant undifferentiated retroperitoneal mass as large B-cell lymphoma. Over a period of $\sim 10$ years, the patient had suffered from three different malignancies. To the best of our knowledge, such a combination of sporadic adenomatous colonic polyposis, colorectal carcinoma and two extra-intestinal non-Hodgkin lymphomas has not been reported to date. It should be considered that each malignancy increases the risk for other neoplastic diseases and a close follow-up is crucial for early detection of second malignancies and neoplastic syndromes.
\end{abstract}

Correspondence to: Dr Seyed Amirhossein Fazeli, Department of Internal Medicine, School of Medicine, Zahedan University of Medical Sciences, 1st Kilometer, Persian Gulf Highway, Zahedan 9816743111, Iran

E-mail: sa.fazeli@gmail.com

Key words: colonic polyposis, lymphoblastic lymphoma, large B-cell lymphoma, adenocarcinoma

\section{Introduction}

Colonic polyposis includes different types of polyps, such as adenomatous, hamartomatous and hyperplastic $(1,2)$. Familial adenomatous polyposis (FAP), Gardner's syndrome, MUTYH-associated polyposis (MAP), familial juvenile polyposis and Peutz-Jeghers syndrome, are colonic polyposis syndromes with different genetic backgrounds and associated symptoms $(1,2)$.

Over several years, the disorders associated with colonic polyposis syndromes were gradually identified $(1,2)$. The extracolonic disorders associated with these syndromes are mainly benign. However, several associated malignancies have also been reported (1-3).

Hematological malignancies have not been described in the original polyposis syndromes $(1,2)$; however, an increasing number of studies highlighted the association between colonic polyposis and hematological malignancies, of either lymphomatous or myeloid origins (4-6).

We herein describe the case of a young male patient with a history of childhood lymphoblastic lymphoma who, 8 years after the initial malignancy, was diagnosed with colonic polyposis, colorectal adenocarcinoma and synchronous retroperitoneal large-B cell lymphoma.

\section{Case presentation}

Our patient was a 22-year-old man from Zahak, located in southeast Iran. In June, 2002, when the patient was 9 years old, he was admitted to the Emergency Department with dyspnea, facial edema, intermittent low-grade fever and productive cough that had appeared 8 days prior to admission. The detailed family history was negative for malignancies, colonic polyposis and other familial syndromes.

On physical examination, the patient was febrile, with respiratory distress and periorbital and facial edema extending to the submandibular area, neck and anterior chest wall. There was 
no cervical lymphadenopathy, but a lymph node sized $2 \times 2 \mathrm{~cm}$ was found in the left axilla. The other physical findings were normal, apart form bilateral hydrocele. The clinical findings were compatible with superior vena cava syndrome.

The laboratory findings were as follows: White blood cell count, 7.8×10\% $/ \mathrm{ml}$; hemoglobin concentration, $8.9 \mathrm{~g} / \mathrm{dl}$; platelet count, 406×10 $/ \mathrm{ml}$; erythrocyte sedimentation rate (ESR), 55; and lactate dehydrogenase (LDH) level, $587 \mathrm{U} / 1$. A chest $\mathrm{X}$-ray revealed mediastinal widening. The abdominal ultrasonography was negative for para-aortic lymphadenopathy and hepatosplenomegaly. A contrast-enhanced chest computed tomography (CT)-scan revealed an anterior mediastinal mass. The abdominopelvic CT-scan was normal. Following a transthoracic incisional biopsy, the mediastinal mass was diagnosed as lymphoblastic lymphoma. The bone marrow aspiration and cerebrospinal fluid analysis were negative.

After 8 days of hospitalization and initial treatment with dexamethasone, vincristine and cyclophosphamide, the patient was discharged with an improved general condition. For 24 months the patient was treated with the Berlin-Frankfurt-Munich non-Hodgkin lymphoma (BFM-NHL) protocol. In February, 2003, maintenance therapy was initiated at week 34 of the protocol, with daily 6-mercaptopurine and weekly oral methotrexate. After 15 months of maintenance therapy, the BFM-NHL protocol was completed in June, 2004. The complete blood count, abdominal and testicular ultrasonography and chest CT scan were normal at the end of the maintenance therapy. Prophylactic cranial irradiation was performed, with 1,800 cGy in 10 sessions over 2 weeks in July, 2003.

In March, 2010, 8 years after the first presentation and when the patient was aged 18 years, he was referred to the Adult Hematology and Medical Oncology service with a history of vague sustained periumbilical abdominal pain and lower gastrointestinal bleeding. The physical examination revealed a soft abdominal mass in the periumbilical area; the other findings were not significant. Given the prolonged interval from the previous lymphoblastic lymphoma diagnosis, a second malignancy was suspected. A contrast-enhanced abdominopelvic CT scan revealed solid masses in the retroperitoneal and intra-abdominal areas.

The exploratory laparotomy revealed numerous mucosal polyps throughout the large intestine, and multifocal masses in the ascending and transverse colon and rectosigmoid region. A retroperitoneal mass was also identified. Total colectomy with ileorectal anastomosis and retroperitoneal tumor resection and lymphadenectomy were performed.

The pathological examination revealed $>100$ tubular adenomatous polyps with a diameter of $\leq 2 \mathrm{~cm}$, and a multifocal, polypoid, well-differentiated adenocarcinoma with a mucinous component. Lymph node involvement and pericolic invasion were detected. However, the appendix and terminal ileum were tumor-free. Furthermore, the immunohistochemical examination confirmed the malignant undifferentiated retroperitoneal mass to be large B-cell lymphoma, positive for CD45 and CD20 and negative for cytokeratin.

The patient received 6 cycles of ifosphamide, etoposide and oxaliplatin (IVOX protocol) from June to November, 2011. The gallium scan confirmed complete remission and surveillance was scheduled from February, 2011 onwards.
Although the surveillance laboratory markers, including ESR, LDH and carcinoembryonic antigen, were negative for recurrence of epithelial and/or lymphomatous malignancies, the follow-up colonoscopy in 2013 reveled an ulcerated tubulovillous adenomatous polyp with high-grade dysplasia. In addition, two tubular adenomatous polyps were found at a distance of 3 and $8 \mathrm{~cm}$ from the anal verge. A subsequent colonoscopy performed 6 months later detected a mass with malignant characteristics in the rectum. The pathological examination revealed an invasive adenocarcinoma in a background of a high-grade villous adenomatous polyp.

The patient underwent local surgical therapy with permanent colostomy. Subsequently, 6 cycles of adjuvant chemotherapy with the FOLFIRI protocol were administered and the patient has been receiving capecitabine and bevacizumab as maintenance therapy, without any relapses to date. The detailed family history was negative for malignancies, colonic polyposis and other familial syndromes.

\section{Discussion}

Colonic polyposis is a heterogeneous group of neoplastic disorders with different characteristics, including adenomatous and hamartomatous polyps $(1,2)$. FAP is an inherited polyposis syndrome characterized by the presence of adenomatous polyps in the colon and rectum (1). The classic syndrome originates from a germline mutation in the adenomatous polyposis coli (APC) gene and the patients are at high risk of colon cancer if left untreated $(1,7)$. A strong family history of colonic polyps and cancer is present (1). Different variants of this syndrome include attenuated FAP, Gardner's syndrome, and MAP $(1,2)$. Familial juvenile polyposis, hyperplastic polyposis and Peutz-Jeghers syndrome are other examples of colonic polyposis, with different origins $(1,2)$. Furthermore, lymphoproliferative disorders may resemble polyposis coli (8).

Different types of colonic polyposis are associated with certain neoplasms $(1,2,9)$. These neoplasms are mainly benign, including desmoid tumors, osteomas and epidermoid cysts $(1,3,9,10)$, but malignancies such as thyroid cancer and upper gastrointestinal adenocarcinoma have also been reported (11-16).

Clinical and genetic data suggest that hematological malignancies are not common extracolonic manifestations in FAP and other colonic polyposis syndromes (6). For example, it has been reported that the mutation of the APC gene may not be the major cause of hematological malignancies (17). However, the number of reports on the association between colonic polyposis and hematological malignancies is increasing. These malignancies are of myelogenous origin, such as acute and chronic myelogenous leukemia, as well as lymphoproliferative disorders (4-6,11).

We reported a unique combination of sporadic adenomatous colonic polyposis, colorectal carcinoma, lymphoblastic lymphoma and large B-cell lymphoma in a young male patient.

The reports of lymphoblastic lymphoma in association with colonic polyposis are limited. Kiratli et al (18) reported a sporadic unilateral retinoblastoma in a 3-year-old boy treated with enucleation, with no adjuvant chemoradiation. Lymphoblastic lymphoma and juvenile hamartomatous polyposis were diagnosed 5 and 6 years later, 
respectively. Kaplan et al (19) reported siblings with hereditary von Recklinghausen's neurofibromatosis and familial lymphoblastic lymphoma. Colonic polyposis and the characteristics of Gardner's syndrome were found in one of the siblings.

Certain studies report synchronous occurrence of colorectal carcinoma and gastrointestinal NHL (20-22). However, co-occurrence of colonic polyposis and colorectal carcinoma with two extraintestinal synchronous and metachronous non-Hodgkin lymphomas is unusual.

Second malignancies are very important when a patient is under surveillance for a primary malignancy. Suspicion should arise when the clinical findings, such as time course, are not fully compatible with relapse of the primary malignancy. A precise schedule of surveillance and patient adherence are crucial for optimal follow-up outcomes.

\section{Acknowledgements}

The authors would like to thank the staff of the Internal Medicine, Surgery and Pathology wards at the Ali-Ebne-Abitaleb Hospital in Zahedan, Iran, for their techniqual support.

\section{References}

1. Half E, Bercovich D and Rozen P: Familial adenomatous polyposis. Orphanet J Rare Dis 4: 22, 2009.

2. Omundsen M and Lam FF: The other colonic polyposis syndromes. ANZ J Surg 82: 675-681, 2012.

3. Groen EJ, Roos A, Muntinghe FL, Enting RH, de Vries J, Kleibeuker JH, Witjes MJ, Links TP and van Beek AP: Extra-intestinal manifestations of familial adenomatous polyposis. Ann Surg Oncol 15: 2439-2450, 2008

4. Itsukuma $\mathrm{T}$, Ishikawa $\mathrm{H}$, Misawa M, Kai S, Fujimori Y, Nakagawa K, Hirota S, Sugihara A, Terada N and Hara H: Familial adenomatous polyposis complicated by chronic myelogenous leukemia: Response to imatinib mesylate. J Gastroenterol 42: 402-405, 2007.

5. Greenberg MS, Anderson KC, Marchetto DJ and Li FP: Acute myelocytic leukemia in two brothers with polyposis coli and carcinoma of the colon. Ann Intern Med 95: 702-703, 1981.

6. Frizelle FA, Hemmings CT, Whitehead MR and Spigelman AD: Familial adenomatous polyposis and duodenal lymphoma. Dis Colon Rectum 46: 1698-1701, 2003.

7. Fearnhead NS, Britton MP and Bodmer WF: The ABC of APC. Hum Mol Genet 10: 721-733, 2001.

8. Rix E, Bröker HJ, Waldherr R and Ho AD: Non-Hodgkin's lymphoma resembling polyposis coli. A case report. Endoscopy 18: 165-167, 1986.
9. Fotiadis C, Tsekouras D, Antonakis P,Sfiniadakis J, Genetzakis M and Zografos GC: Gardner's syndrome: A case report and review of the literature. World J Gastroenterol 11: 5408-5411, 2005.

10. Soravia C, Berk T, McLeod RS and Cohen Z: Desmoid disease in patients with familial adenomatous polyposis. Dis Colon Rectum 43: 363-369, 2000.

11. Murphy H, Taylor W, Ellis A and Sturgess R: An unusual case of Turcot's syndrome associated with ileal adenocarcinoma, intestinal non-Hodgkin's lymphoma and duodenal adenocarcinoma. Review of the classification and genetic basis of Turcot's syndrome. Fam Cancer 4: 139-143, 2005.

12. Giardiello FM, Offerhaus GJ, Lee DH, Krush AJ, Tersmette AC, Booker SV, Kelley NC and Hamilton SR: Increased risk of thyroid and pancreatic carcinoma in familial adenomatous polyposis. Gut 34: 1394-1396, 1993.

13. Jagelman DG, DeCosse JJ and Bussey HJ: Upper gastrointestinal cancer in familial adenomatous polyposis. Lancet 1: 1149-1151, 1988.

14. Spigelman AD, Williams CB, Talbot IC, Domizio P and Phillips RK: Upper gastrointestinal cancer in patients with familial adenomatous polyposis. Lancet 2: 783-785, 1989.

15. Soravia C, Sugg SL, Berk T, Mitri A, Cheng H, Gallinger S, Cohen Z, Asa SL and Bapat BV: Familial adenomatous polyposis-associated thyroid cancer: A clinical, pathological and molecular genetics study. Am J Pathol 154: 127-135, 1999.

16. Matsuo H, Kato T, Hirai T, Kodera Y, Kito T and Nakamura H: A case of cancerous familial adenomatous polyposis in urinary bladder due to migration of colonic mucosa through rectovesical fistula. Am J Gastroenterol 95: 1352-1354, 2000

17. Wada M, Miller CW, Yokota J, Lee E, Mizoguchi H and Koeffler HP: Molecular analysis of the adenomatous polyposis coli gene in sarcomas, hematological malignancies and noncolonic, neoplastic tissues. J Mol Med (Berl) 75: 139-144, 1997.

18. Kiratli H, Bilgiç S and Ozerdem U: Retinoblastoma with acute lymphoblastic leukemia, polyposis coli and multiple hamartomas. J AAPOS 2: 385-386, 1998.

19. Kaplan J, Cushing B, Chang CH, Poland R, Roscamp J, Perrin E and Bhaya N: Familial T-cell lymphoblastic lymphoma: Association with Von Recklinghausen neurofibromatosis and Gardner syndrome. Am J Hematol 12: 247-250, 1982.

20. Genovese F, Becchina G, Nagar C, Ottoveggio G, Giacalone B, Scaglione G, Varriale E and Tralongo V: Primary diffuse large B-cell lymphoma developing within a rectal tubular adenoma with low-grade dysplasia: A case report. J Med Case Rep 8: 103, 2014.

21. Song W, He YL, Han FH, Cai SR and Peng JJ: Rectal non-Hodgkin lymphoma with concomitant rectal adenocarcinoma: A case report and literature review. Chin J Gastrointest Surg 14: 617-619, 2011 (In Chinese).

22. Sasaki S, Hatanaka K, Sahara N, Uekusa T, Hirayama K, Shirahata A and Ishimaru M: Collision tumor of primary malignant lymphoma and adenocarcinoma in the colon: Report of a case. Surg Today 40: 975-981, 2010. 